Full Length Article

\title{
Effects of manipulating the number of targets in U9, U11, U15 and U17 futsal players' tactical behaviour
}

\author{
Bruno Travassos $^{\mathrm{a}, \mathrm{c}, *}$, Diogo Coutinho ${ }^{\mathrm{b}, \mathrm{c}}$, Bruno Gonçalves ${ }^{\mathrm{b}, \mathrm{c}}$, Paulo Pedroso ${ }^{\mathrm{a}}$, \\ Jaime Sampaio ${ }^{\mathrm{b}, \mathrm{c}}$ \\ a Department of Sports Sciences, University of Beira Interior, Covilhã, Portugal \\ ${ }^{\mathrm{b}}$ Department of Sports Sciences, Exercise and Health, University of Trás-os-Montes and Alto Douro, Vila Real, Portugal \\ ${ }^{\mathrm{c}}$ CIDESD - Research Center in Sports, Health Sciences and Human Development, CreativeLab, Portugal
}

\section{A R T I C L E I N F O}

\section{Keywords:}

Futsal

Age-related experience

Small-sided games

Task constraints

Positional variables

\begin{abstract}
A B S T R A C T
This study identified how the manipulation in the number of goal targets affects the tactical behaviour of players from different age groups (U9, U11, U15 and U17). Forty youth futsal players performed two small-sided games based on Gk + 4vs4 + Gk situation with one regular and two small targets. TACTO software was used to capture players and ball displacements. The following variables were computed and presented as absolute values, coefficient of variation and regularity (approximate entropy): (i) distance from each player to the team centre (DtC); (ii) distance from each player to the ball (DtB); (iii) distance between team centres (DbTC); and (iv) distance from team centre to the ball (DCtB). The team dispersion increased with two goal targets (mainly the DbTC, U9 and U17, large effects; U11 and U15, very large effects). Also, the use of two goal targets condition increased the variability in the DbTC (U9, small effects; U11 and U15 moderate effects; U17, very large effects) and DCtB (U9 and U17 moderate effects and U11 and U15 small effects). Overall, the approximate entropy values showed higher regularity in the condition with two targets. All age groups were sensitive to the manipulation of goal targets, however, the U9 were the most sensitive to the changes, as seen by the dispersion of players in the field. Thus, coaches can use one target to promote movement irregularity of players and two targets to increase the team dispersion, mainly in younger age groups that tend to be agglomerated around the ball.
\end{abstract}

\section{Introduction}

In team sports, such as futsal, the manipulation of task constraints in small-sided and conditioned games (SSCG) seems to be an effective approach to skill acquisition (Coutinho et al., 2018; Davids, Araújo, Correia, \& Vilar, 2013; Sgrò, Bracco, Pignato, \& Lipoma, 2018). In addition, these manipulations expose the players to a wide range of scenarios leading to the emergence of adaptive behaviours that are required during the competitive demands (Gonçalves, Marcelino, Torres-Ronda, Torrents, \& Sampaio, 2016; Seifert, Button, \& Davids, 2013). Indeed, adaptive behaviours in team sports result from the interactions that each subject create with teammates, the opponents and the surrounding environment leading to the emergence of opportunities for action (Coutinho et al., 2018; Fajen, 2007; Travassos, Araujo, Duarte, \& McGarry, 2012; Travassos, Duarte, Vilar, Davids, \& Araújo, 2012). This evidence suggests that training tasks should maintain similarities in informational constraints that support the spatial-temporal relations between teammates and opponents in relation to the field and goal target location (Laakso, Travassos, Liukkonen, \& Davids, 2017). In

\footnotetext{
* Corresponding author at: Department of Sport Sciences, University of Beira Interior, Convento de Sto. António, 6201-001 Covilhã, Portugal.

E-mail address: bruno.travassos@ubi.pt (B. Travassos).
} 
line with that, coaches should manipulate the training tasks to highlight some informational constraints that promotes the players' adaptation to the specific demands of competition (Fajen, 2007; Gonçalves, Marcelino, et al., 2016; Travassos, Araujo, et al., 2012). Therefore, it assumes a major relevance knowing which tasks constraints guide players to better exploit collective possibilities for action according to their age or skill level (Davids et al., 2013; Gonçalves, Esteves, Folgado, Ric, Torrents, \& Sampaio, 2016).

The pedagogical interest on understanding the manipulation of task constraints to boost the learning and performance in team sports has grown exponentially (Davids et al., 2013; Mitchell, Oslin, \& Griffin, 2013; Sgrò et al., 2018). For example, it was well reported that decreasing the number of players induce higher training intensities and technical demands (Hill-Haas, Dawson, Impellizzeri, \& Coutts, 2011). However, most of the available studies neglect how the different task constraints affects the tactical behaviour of players and teams (Hill-Haas et al., 2011).

Over the last years, there have been some attempts to understand how players adapt their tactical behaviour according to the manipulation of different task constraints during SSCG (Sampaio, Lago, Gonçalves, Maçãs, \& Leite, 2014; Silva et al., 2014). For instance, Travassos, Gonçalves, Marcelino, Monteiro, and Sampaio (2014) studied the effects of the amplification of the number of goal targets from 2 (with goalkeeper) to 6 (small goals), and concluded that with 6 targets there was an increase in the distance between the opposing teams, as well as, in the time that each team spent in lateral corridors of the pitch. Also, Gonçalves, Esteves, et al. (2016) showed in a recent study that players interpersonal coordination tendencies between teammates and the players' physical performance decreased when the players were constrained according to specific pitch zones compared to the free condition. Overall, these studies highlighted that particular behaviours emerged based on the information amplified by each task constrain but few of them have reference to the changes promoted on players of different age or level of practice.

Accordingly, it is only known that older teams revealed: (a) higher team dispersion on the pitch, as well as a higher regularity in the collective behaviour than younger ones (Barnabe, Volossovitch, Duarte, Ferreira, \& Davids, 2016; Folgado, Lemmink, Frencken, \& Sampaio, 2014; Olthof, Frencken, \& Lemmink, 2015); (b) the type of scoring mode (line goal target, double goal target or central goal target) constraint defensive performance of players from different age groups (under-13 and under-15) (Almeida, Duarte, Volossovitch, \& Ferreira, 2016); and (c) field dimension or numerical relations constraint players' tactical behaviour of players from different skill levels (regional or national level) (Silva et al., 2014).

Overall, the available research revealed that players are likely to exhibit different movement behaviours based on changes in task constraints and these behaviours are also constrained by the age of the players. Although these insightful advancements, most of the research conducted on the manipulation of task constraints and players' ages have been developed in football. In fact, the number of youth futsal practitioners has been considerably increasing and it is important that coaches understand how to design appropriate learning environments that help the players to develop tactical behaviour and promote more adaptive behaviours according to changes in game environment (Davids et al., 2013). In addition, most of the studies in this domain have only compared two age groups, and a better understanding of the effects of certain task constraints in different age groups will be ensured if more age groups are considered (Davids et al., 2013; Serra-Olivares, González-Víllora, García-López, \& Araújo, 2015).

Thus, this study aimed to identify how the manipulation in the number of goal targets affects the tactical behaviour of players belonging to different age groups (U9, U11, U15 and U17) during a 4vs4 SSCG. For this purpose, it was measured the concentration / dispersion of the teams, and also processed the variability and regularity of the players' behavioural patterns during the tasks. It was hypothesized that different ages groups will reveal different tactical behaviours according to the different number of goal targets used, with an increase in the space used and in the variability of behaviours for two goal targets in relation to one goal target and a general increase on the space used for older players. In fact, using two goal targets requires a wide attention deployment and defocused attention. Also, and based on previous research (Barnabe et al., 2016; Folgado et al., 2014; Olthof et al., 2015), it was also expected higher regularity of behaviours of older players. It was expected that the results from this study might help to design training tasks more appropriate for each developmental age.

\section{Methods}

\subsection{Participants}

The sample was composed by forty futsal players from four age groups (under-9 (U9), $n=10$, mean age $7.3 \pm 0.78$ years; under11 (U11), $n=10$, age $9.6 \pm 0.48$ years; under-15 (U15), $n=10$, age $12.5 \pm 1.03$ years; under-17 (U17), $n=10$, age $15.5 \pm 0.5$ years) defined according to competitive rules of the national football federation. All the players were part of the same club, participated in two training sessions per week ( $~ 90$ min per session) and played an official game during the weekend. Although the goalkeepers participated in the study, they were excluded from the data analysis, as the goalkeepers' positioning is very restricted to a specific pitch area and their game dynamics different from the outfield players. An informed consent was provided to the coaches, players, and their parents, as well as by the club, before the beginning of the study. All participants were notified that they could withdraw from the study at any time. The experimental procedures were approved by the local Institutional Research Ethics Committee and followed the guidelines stated in the Declaration of Helsinki.

\subsection{Procedures}

All players were familiarized with the SSCG conditions one week before the testing session during the regular training. Afterwards, each age group performed two testing sessions where in each session was performed one of the SSCG conditions (1 Goal targets or 2 Goal targets, see Fig. 1). The head coach of each age group selected the ten best players from the team, according to their 


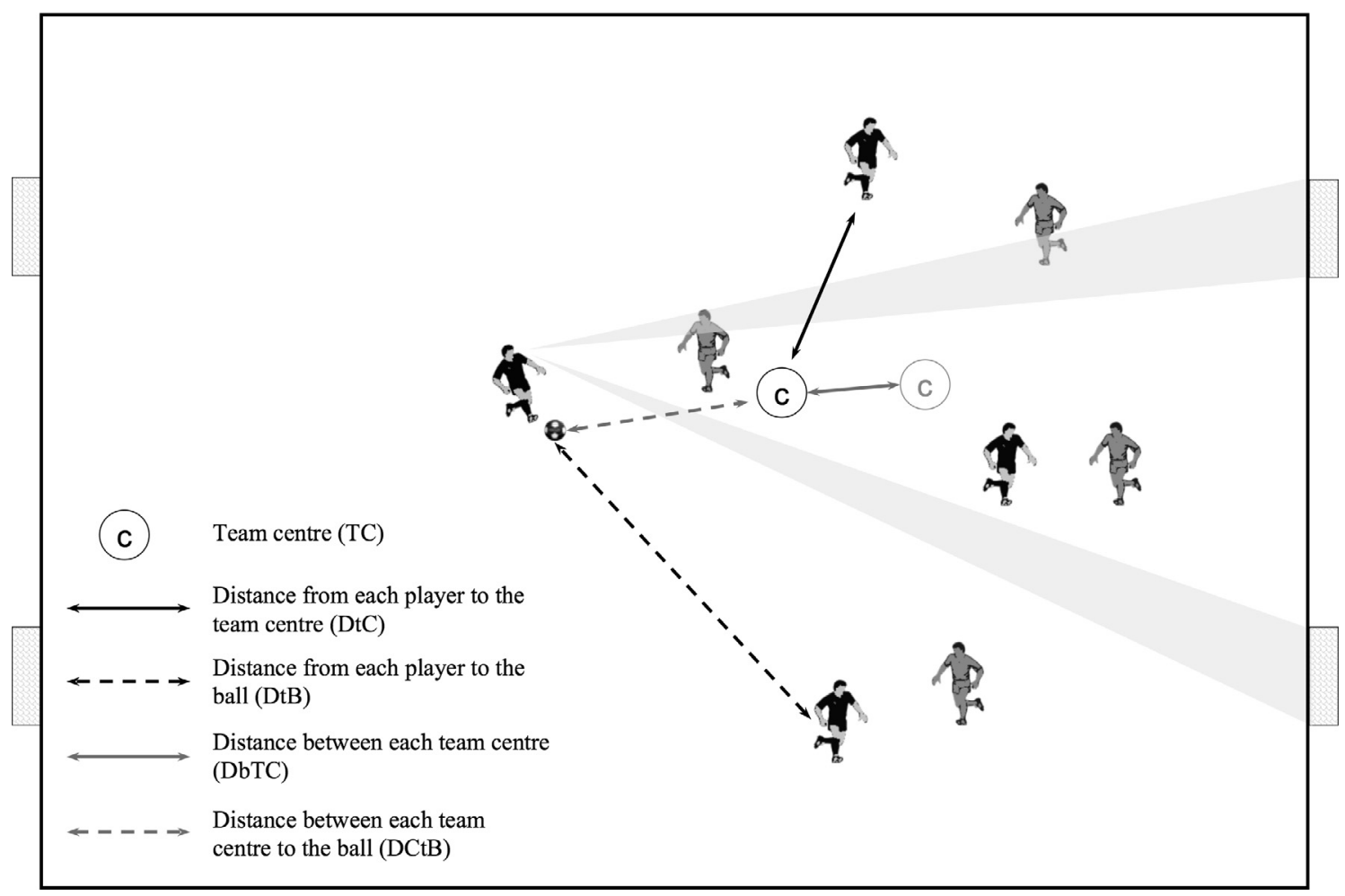

Fig. 1. Representation of the computed variables for the players and teams' tactical behaviour.

technical, tactical and physical skills and then divided them into two balanced teams (Casamichana \& Castellano, 2010). Apart from the goalkeepers, no positional roles were considered as all outfield players were required to freely move at will through the pitch. Before the SSCG, there was a standardized 10-min warm-up based on running and a ball possession game (5-a-side without goal targets). The games were played on a futsal pitch with $38 \times 18 \mathrm{~m}$ (width $\times$ length) according to two SSCG conditions: (i) $\mathrm{Gk}+4 \mathrm{v} 4+$ Gk with two official scoring goal targets $(3 \times 2 \mathrm{~m})$, (1 Goal target); and (ii) $4 \mathrm{v} 4$ with four small goal targets $(3 \times 1.55 \mathrm{~m})$ placed in the end line and distanced from each other from $9 \mathrm{~m}$ (2 Goal targets). Each SSCG was based on three bouts of 5-min interspersed with a 5-min of passive recovery and performed in two non-consecutive days. Therefore, each age group participated in a total of 6 SSCG bouts, during the normal training sessions at the same time of the day. The SSCG were performed with the official FIFA futsal rules. Several balls were placed around the pitch to reduce the time lost with the ball out of play. Also, no coach feedback or encouragement was permitted to avoid possible interferences in the players' behaviours.

\subsection{Data collection and analysis}

All the SSCG were recorded using a digital video camera (Gopro Hero 3, HD 3.03.03.00), at 25 Hz, with a resolution of $1920 \times 1080$ pixels positioned in a superior plane ( $5 \mathrm{~m}$ of height) and located $45^{\circ}$ from one of the goal lines. This position was used considering that it allowed to capture all the pitch. The TACTO 8.0 software was used to capture the bi-dimensional (2D) virtual movements coordinates from each player and the ball (Fernandes, Folgado, Duarte, \& Malta, 2010). The players were tracked using a mouse cursor to follow the middle point between the feet of each player in all video clips (Vilar, Duarte, Silva, Chow, \& Davids, 2014). This point was considered based on the assumption that it may represent the player centre of mass on the pitch (Duarte et al., 2010; Vilar, Duarte, et al., 2014). The digitizing of the ball consisted of tracking the vertical projection of the ball on the playing surface in slow video motion using the computer cursor (Duarte et al., 2010). Afterwards, the virtual coordinates from the players were converted into the pitch real coordinates (meters) using a bi-dimensional direct linear transformation (2D-DLT) method (Duarte et al., 2010). Then, these coordinates were exported and computed using appropriate routines in Matlab ${ }^{\circledR}$ (MathWorks, Inc., Massachusetts, USA) and the associated tracking error noise from the manual digitalization was reduced by smoothing the data based on a $6 \mathrm{~Hz}$ Butterworth low pass filter (Duarte et al., 2010).

Five players from each team and the ball were randomly selected and the data trajectories were re-digitized by the same researcher to calculate coefficient of reliability (R) and technical error of measurement (TEM) (Goto \& Mascie-Taylor, 2007). The TEM yielded values of $0.21 \mathrm{~m}(1.77 \%)$ and $0.26 \mathrm{~m} \mathrm{(2.29 \% )} \mathrm{for} \mathrm{players} \mathrm{and} \mathrm{ball,} \mathrm{respectively.} \mathrm{The} \mathrm{coefficient} \mathrm{of} \mathrm{reliability} \mathrm{revealed} \mathrm{good}$ reliability for players $(\mathrm{R}=0.97)$ and for ball $(\mathrm{R}=0.95)$ trajectories.

The dynamic positional data of the players and the ball were used to determine the following individual variables: (i) distances to the team centre (DtC); (ii) distance to the ball (DtB). The following collective variables were also computed: (i) distance between the 
two-team's centre (DbTC); (ii) and distance between each team centre to the ball (DCtB). All the previous variables were dynamically calculated and computed for each timeframe (25) along the time of each game condition. The length of the timeseries used to process each variable was 7500 data sets ( 5 min of task duration $\times 60 \mathrm{~s} \times 25 \mathrm{~Hz}$ ). Afterwards, for individual variables it was computed and presented in absolute values (meters), variability (expressed by the coefficient of variation, CV) and predictability (expressed by the approximate entropy values, ApEn). For the collective variables, the absolute values, variability and predictability were computed in blocks of 1-minute length.

ApEn technique was used to assess regularity or predictability of the variables time series. Input values for computations were 2.0 to the vector length (m) and 0.2 standard deviations to the tolerance factor (r) (Stergiou, Buzzi, Kurz, \& Heidel, 2004). The outcome range between 0 and 2 (arbitrary units) and lower values represented more repeatable, regular, predictable and less chaotic sequences of data points (Pincus, 1991; Stergiou et al., 2004). From a processing approach, ApEn expresses the probability that the configuration of one segment of data in a time series will allow the prediction of the configuration of another segment of the time series a certain distance apart (Harbourne \& Stergiou, 2009). This technique permits to identify if displacement trajectories express a regular and predictable pattern which may, in turn, provide information regarding their tactical behaviour (Gonçalves, Esteves, et al., 2016; Sampaio \& Macas, 2012)

\subsection{Statistical analysis}

Magnitude-based inferences and precision of estimation were used to analyse the data (Batterham \& Hopkins, 2006). Prior to the scenario comparisons for each age groups (i.e. 1 Goal target vs. 2 Goal targets), all processed variables were log-transformed to reduce the non-uniformity of error. A descriptive analysis was performed using mean and standard deviations for each variable and presented graphically (the mean shown is the back-transformed mean of the log transform). The mean and standard deviation for individual variables were computed considering each bout duration ( 3 bouts $\times 4$ players $\times 2$ teams, $N=24$ for each age) and for collective variables were computed in blocks of 1 -minute length ( 3 bouts $\times 5$-min $\times 2$ teams, $N=30$ for each age). Differences in means for both pairs of scenarios were also expressed and graphically represented in percentage units with $90 \%$ confidence limits (CL). The effect was reported as unclear if the CL overlapped the thresholds for smallest worthwhile changes, which were computed from the standardized units multiplied by 0.2. Magnitudes of clear effects were described probabilistic according to the follow scale: $<0.5 \%$, most unlikely; 0.5-5\%, very unlikely; 5-25\%, unlikely; 25-75\%, possibly; 75-95\%, likely; 95-99.5\%, very likely; > 99.5\%, most likely (Hopkins, Marshall, Batterham, \& Hanin, 2009). The comparisons among game scenarios were also assessed via standardized mean differences with 90\% confidence intervals (Cumming, 2012; Hopkins et al., 2009). Thresholds for effect sizes statistics were 0.2, trivial; 0.6, small; 1.2, moderate; 2.0, large; and > 2.0, very large (Hopkins et al., 2009).

\section{Results}

Fig. 2 represents the descriptive analysis for the considered variables according to the game conditions in each age group, while the Fig. 3 presents the standardized Cohen differences. Increasing the number of goal targets from one to two goal targets revealed a likely increase in the DtC for the U9 age group (small effects), a likely increase for the DtB for the U9 and U15 age groups and a possible increase for the U17 age group (small effects, Fig. 3). The DbTC variable shown higher consistency in the results between the different age groups. The increase from one to two goal targets showed a most likely increase in the DbTC for all age groups (U9 and U17, large effects; U11 and U15, very large effects). The analysis of the coefficient of variation showed higher values for most of the studied variables in the condition with two goal targets, with the exception of the DtC for the U15 and U17 age groups. There was a most likely increase in the coefficient of variation for the DtC for the U9 age group (moderate effect) and most likely decrease in the U15 age group (moderate effect) when compared one with two goal targets. The coefficient of variation for the DbTC variable revealed a most likely increase for U11 and U17 age groups, a very likely increase for the U15 and a likely increase for the U9 age group (U9, small effects; U11 and U15 moderate effects; U17, very large effects). Increasing the number of goal targets from one to two goal targets presented a very likely increase in the coefficient of variation in the DCtB for the U9 and U17 age groups (moderate effects) and a likely increase for the U11 and U15 (small effects).

Overall, the results from the approximate entropy values showed higher regularity in the condition with two goal targets, with the exception of the U15 age group for the DtC that evidenced higher regularity in the condition with one goal target. The increase of the number of goal targets from one to two showed a most likely increase in the regularity in the DtC and DtB for the U9 and U17 age groups (DtC, moderate effects; DtB, large effects), a very likely increase for the DtB in the U15 age group (moderate effect) and a likely increase for the DtB in the U11 age group (moderate effect). Also, increasing the number of goal targets from one to two showed a very likely increase in the DbTC for the U9 and U11 (U9, large effects; U11, moderate effects), and a likely increase for the U17 (small effect). Finally, the condition with two goal targets evidenced a most likely increase in the DCtB for the U9 age group (large effect), and a very likely increase in the U11, U15 and U17 age groups (moderate effects).

\section{Discussion}

This study aimed to measure how the manipulation in the number of goal targets affects the tactical behaviour of players belonging to different age groups (U9, U11, U15 and U17) during futsal SSCG. In line with our expectations, the manipulation of the number of goal targets seems to have a large effect in the player's DbTC, as this variable was sensitive to changes in the task constraint for all the considered age groups. These results are in line with the available literature, which shown that for all age groups, the 

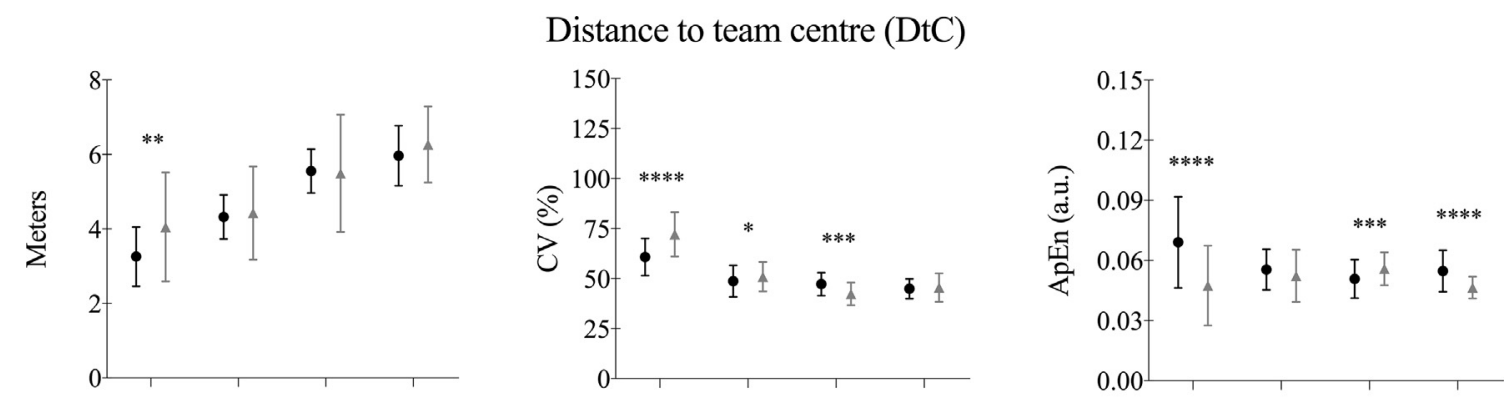

Distance from each player to the ball (DtB)
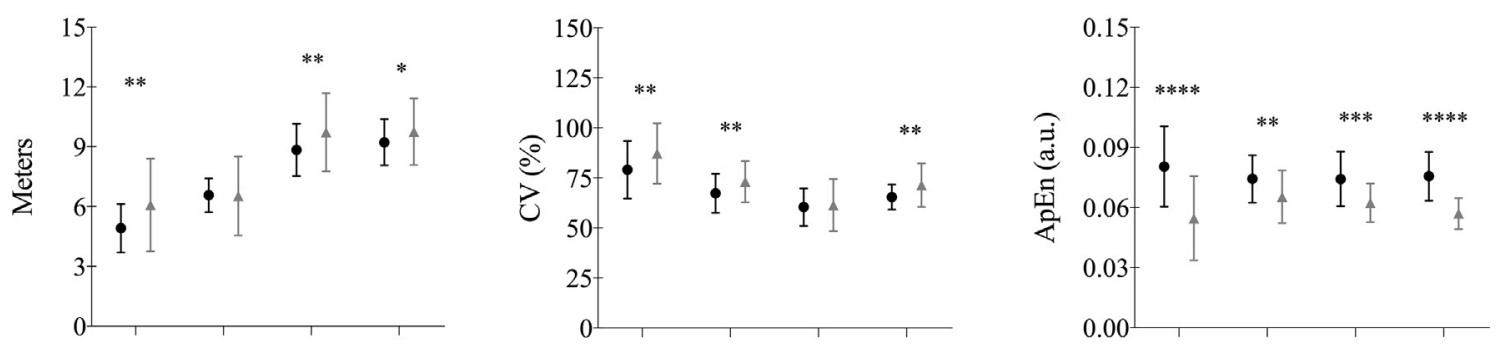

Distance between each team centre (DbTC)
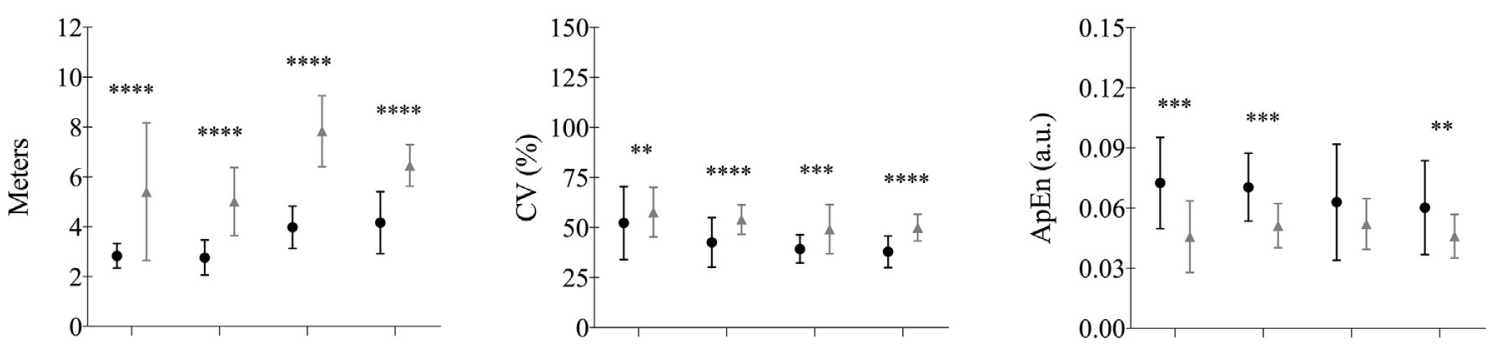

Distance between each team centre to the ball (DCtB)

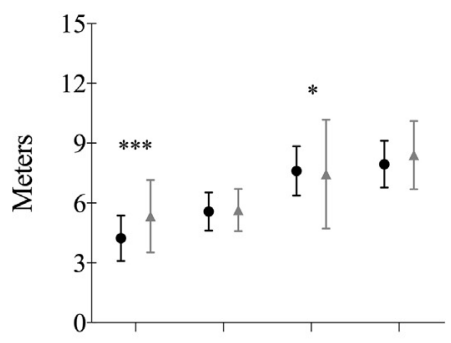

U9 U11 U15 U17

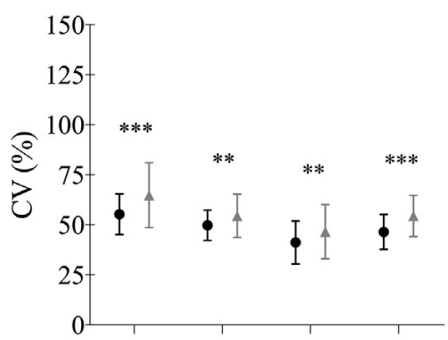

U9 U11 U15 U17

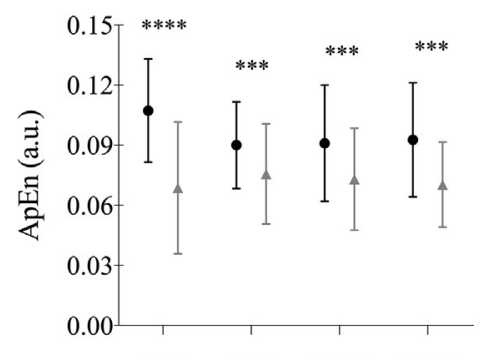

U9 U11 U15 U17

Fig. 2. Mean and standard deviation to absolute values (meters), coefficient of variation (\%CV) and approximate entropy (a.u.) for the considered variables. The likelihood of variation between the two conditions (1 Goal target vs 2 Goal targets) were presented by * with the following legend: *25-75\%, possible; ** 75-95\%, likely; *** 95-99\%, very likely; **** > 99\%, most likely.

distance between teams increase as the number of goal targets increases (Travassos et al., 2014). Similarly, there was also an increase in the DCtB with two goal targets. Thus, independently of the age and experience, the increase in the number of goal targets promote a higher team dispersion in relation to the ball position as well as to the team centre. Interestingly, the U9 was the only age group that showed effects in all considered variables, suggesting the number of goal targets as a sensitive game boundary condition that should be considered when designing training tasks. Accordingly, it promotes dispersion of futsal players in the field and decrease the agglomeration around the ball, which is a typical behaviour from the U9 age group. From the obtained results, it is worth noting that the same constraint did not promote the same effect among age groups. Thus, despite of the increasing amount of research conducted to better understand the effects of the manipulation of the task constraints, further research is required to better understand these effects according to different age groups, allowing to stablish normative guidelines for further game-teaching frameworks (Folgado 

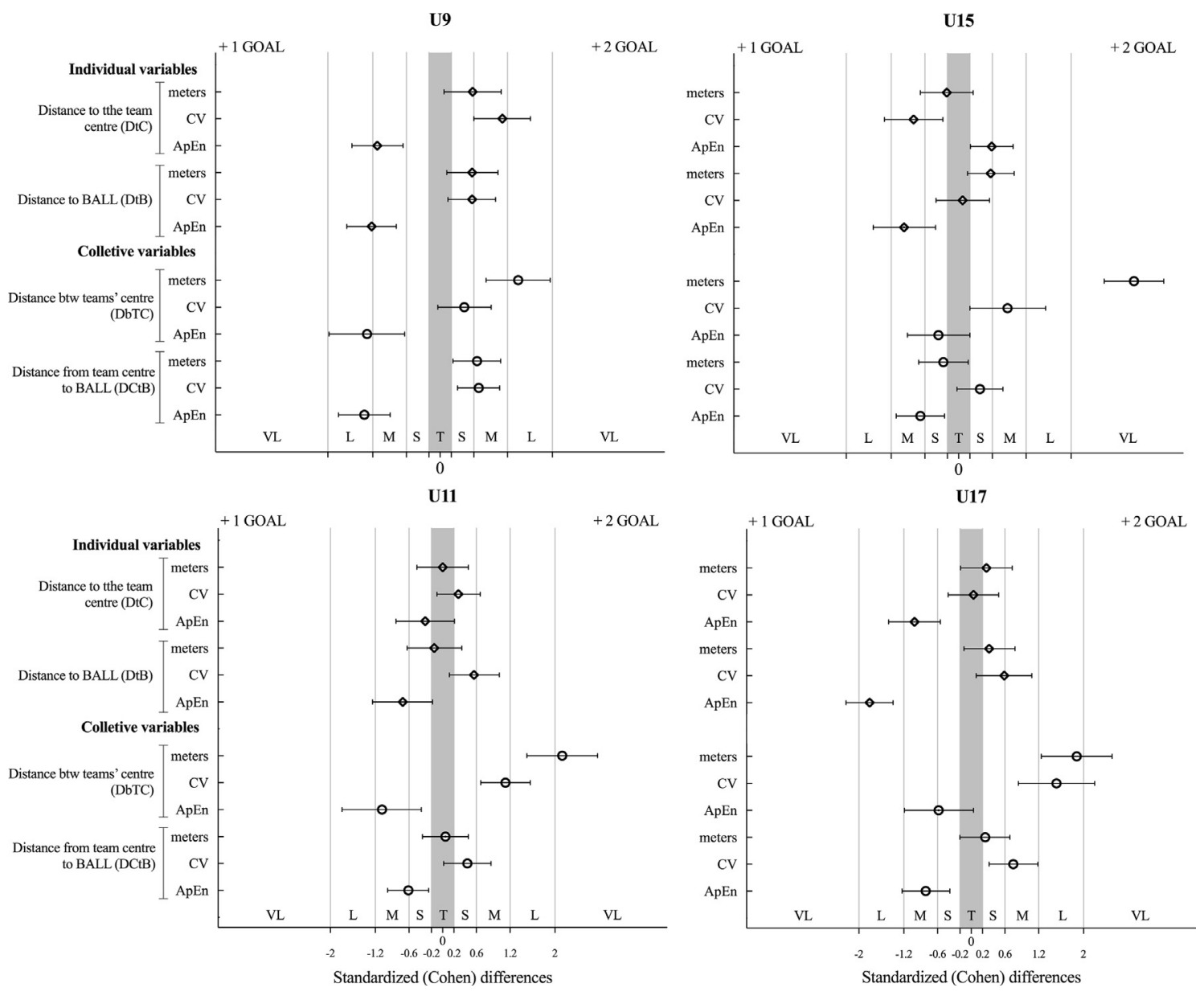

Fig. 3. Standardized (Cohen) differences according to number of targets (1 Goal target vs 2 Goal targets) and respective age group. Error bars indicate uncertainty in the true mean changes with $90 \%$ confidence intervals. $\mathrm{CV}=$ coefficient of variation; ApEn $=$ Approximate Entropy; $\mathrm{VL}=$ very large; $\mathrm{L}=$ large; $\mathrm{M}=$ moderate; $\mathrm{S}=$ small; $\mathrm{T}=$ trivial.

et al., 2014).

The variability (CV) and regularity (ApEn) results revealed higher magnitude of variability and structural regularity of behaviours in the condition with two goal targets for most of the age groups. Based on that, increasing the number of goal targets from one to two, lead to the emergence of more stable positioning behaviours for each team.

Accordingly, with the additional target there was a higher dispersion in the pitch, which combined with an extra option to score may have empathised more stable and regular positioning, as the players might find opportunities to shoot more easily. In fact, previous reports have shown that players evidence more regular positioning when spatial references, such as goal targets, are added to the pitch (Gonçalves et al., 2017). In turn, when there is only one goal target available, the team in possession may have to move more often to create space, decreasing the regularity in the players' movement patterns. Thus, using two goal targets amplifies the information of shooting lines and promoted the adjustment of collective behaviours of attacker and defensive teams (Travassos et al., 2014). Furthermore, despite of the more structured pattern of play and dispersion with two goal targets, the players seem to be able to adapt to the ever-changing configurations of play. In fact, the additional goal target increased the distances variability. The variability may result of the individual necessity of each player and team to constantly adjust his behaviour to higher number of possibilities of opponent team to reach shooting lines. That is, players and teams must be fine-tuned with the spatial-temporal relations with opponents to open or restrict space (Gonçalves, Esteves, et al., 2016). Thus, the increase in the number of goal targets seems to promote higher flexibility of individual and collective behaviours of teams to the dynamic competitive environment (Olthof et al., 2015; Seifert et al., 2013). In contrast, the reduction in the number of goal targets, seems to afford the teams to exhibit behaviours with less variability but with higher irregularity in the spatial occupation (Travassos et al., 2014). Accordingly, the futsal has evolved to a game without positions, where the players are required to constantly move to create unbalance in the opponents' defence. Therefore, the increased movement irregularity during the condition with one goal target may be viewed as the attempts of the teams to break the symmetry with the opposing team (Vilar, Araujo, Travassos, \& Davids, 2014). Based on these results, using two goal targets may be a useful constraint to promote stability on futsal players' moves with a high contrast between defensive and offensive behaviours (Travassos et al., 2014), whereas, conditions with one goal target, due to the reduction on the number of 
possibilities to shoot at goal target promotes more variability on futsal players' moves with a consequent decrease on the distance between teams (Travassos, Duarte, et al., 2012).

Nevertheless, it should be noticed that this study presents some limitations such as a reduced sample and the lack of a classification of players' skills in each age group. However, it reveals an interesting tendency that coaches should account at the time of creating SSCG for their team. Further studies should consider larger samples, including more teams from different levels and characterization of players' skill level. In addition, it may be possible that different physical and technical outcomes may be imposed by the different task constraints applied in this study, as well as according to the age group. Therefore, future studies should also aim to include these domains to provide a better understanding on how players' shape their behaviours according to changes in task boundary conditions.

In practical terms, generally for all the age groups, coaches can use only one goal target to promote team behaviour variability and unpredictable and creative individual movements or two goal targets to promote team dispersion and teams' stability on team behaviour. Overall, these results highlight that coaches should design training tasks that increase the futsal players' ability to explore their boundaries and novel movement solutions for goal achievement according to each age group capabilities

\section{Conclusion}

The results from this study highlighted that different tactical behaviours emerged as result of how the different age groups interacted with the different information emphasized by the manipulation of the number of goal targets. Accordingly, using only one goal target afforded an increase in the behaviour irregularity. In this sense, this task could be used to develop higher unpredictable movements in balanced game environments. In contrast, using two goal targets boosted the team dispersion, as well as higher behavioural regularity that contrasts defensive and offensive behaviours. Additionally, the present study also allowed to identify the U9 age group as the most sensitive group to the changes in the task constraint, promoting a dispersion of players in the field. Thus, the amplification in the information available induced by the increase in the number of goal targets from one to two showed to be useful to produce adaptive movement behaviours in different age groups.

\section{Acknowledgments}

This work was supported by the Research Project NanoSTIMA: Macro-to-Nano Human Sensing: Towards Integrated Multimodal Health Monitoring and Analytics, NORTE-01-0145-FEDER-000016, Fundo Europeu de Desenvolvimento Regional (FEDER) - NORTE 2020 .

\section{Appendix A. Supplementary data} 017.

Supplementary data associated with this article can be found, in the online version, at https://doi.org/10.1016/j.humov.2018.06.

\section{References}

Almeida, C. H., Duarte, R., Volossovitch, A., \& Ferreira, A. P. P. (2016). Scoring mode and age-related effects on youth soccer teams' defensive performance during small-sided games. Journal of Sports Sciences, 34(14), 1355-1362. http://dx.doi.org/10.1080/02640414.2016.1150602.

Barnabe, L., Volossovitch, A., Duarte, R., Ferreira, A. P., \& Davids, K. (2016). Age-related effects of practice experience on collective behaviours of football players in small-sided games. Human Movement Science, 48, 74-81. http://dx.doi.org/10.1016/j.humov.2016.04.007.

Batterham, A. M., \& Hopkins, W. G. (2006). Making meaningful inferences about magnitudes. International Journal of Sports Physiology and Performance, 1(1), 50-57.

Casamichana, D., \& Castellano, J. (2010). Time-motion, heart rate, perceptual and motor behaviour demands in small-sides soccer games: Effects of pitch size. Journal of Sports Sciences, 28(14), 1615-1623.

Coutinho, D., Gonçalves, B., Wong, D. P., Travassos, B., Coutts, A. J., \& Sampaio, J. (2018). Exploring the effects of mental and muscular fatigue in soccer players' performance. Human Movement Science, 58, 287-296. http://dx.doi.org/10.1016/j.humov.2018.03.004.

Cumming, G. (2012). Understanding the new statistics: Effect sizes, confidence intervals, and meta-analysis. Routledge: Taylor \& Francis Group.

Davids, K., Araújo, D., Correia, V., \& Vilar, L. (2013). How small-sided and conditioned games enhance acquisition of movement and decision-making skills. Exercise and Sport Sciences Reviews, 41(3), 154-161.

Duarte, R., Araujo, D., Fernandes, O., Fonseca, C., Correia, V., Gazimba, V., ... Lopes, J. (2010). Capturing complex human behaviors in representative sports contexts with a single camera. Medicina (Kaunas), 46(6), 408-414.

Fajen, B. R. (2007). Affordance-based control of visually guided action. Ecological Psychology, 19(4), 383-410. http://dx.doi.org/10.1080/10407410701557877.

Fernandes, O., Folgado, H., Duarte, R., \& Malta, P. (2010). Validation of the tool for applied and contextual time-series observation. International Journal of Sport Psychology, 41(Suppl. 4), 63-64.

Folgado, H., Lemmink, K. A., Frencken, W., \& Sampaio, J. (2014). Length, width and centroid distance as measures of teams tactical performance in youth football. European Journal of Sport Science, 1(S1), S487-492. http://dx.doi.org/10.1080/17461391.2012.730060 14 Suppl.

Gonçalves, B., Esteves, P., Folgado, H., Ric, A., Torrents, C., \& Sampaio, J. (2016). Effects of pitch area-restrictions on tactical behavior, physical and physiological performances in soccer large-sided games. The Journal of Strength \& Conditioning Research. http://dx.doi.org/10.1519/JSC.0000000000001700 Publish Ahead of Print.

Gonçalves, B., Esteves, P., Folgado, H., Ric, A., Torrents, C., \& Sampaio, J. (2017). Effects of pitch area-restrictions on tactical behavior, physical, and physiological performances in soccer large-sided games. Journal of Strength and Conditioning Research, 31(9), 2398-2408. http://dx.doi.org/10.1519/JSC.0000000000001700.

Gonçalves, B., Marcelino, R., Torres-Ronda, L., Torrents, C., \& Sampaio, J. (2016). Effects of emphasising opposition and cooperation on collective movement behaviour during football small-sided games. Journal of Sports Sciences, 34(14), 1346-1354. http://dx.doi.org/10.1080/02640414.2016.1143111.

Goto, R., \& Mascie-Taylor, C. G. N. (2007). Precision of measurement as a component of human variation. Journal of Physiological Anthropology, 26(2), 253-256. http:// dx.doi.org/10.2114/jpa2.26.253.

Harbourne, R. T., \& Stergiou, N. (2009). Movement variability and the use of nonlinear tools: Principles to guide physical therapist practice. Physical Therapy, 89(3), 
267.

Hill-Haas, S. V., Dawson, B., Impellizzeri, F. M., \& Coutts, A. J. (2011). Physiology of small-sided games training in football: A systematic review. Sports Medicine, 41(3), 199-220. http://dx.doi.org/10.2165/11539740-000000000-00000.

Hopkins, W. G., Marshall, S. W., Batterham, A. M., \& Hanin, J. (2009). Progressive statistics for studies in sports medicine and exercise science. Medicine and Science in Sports and Exercise, 41(1), 3-12.

Laakso, T., Travassos, B., Liukkonen, J., \& Davids, K. (2017). Field location and player roles as constraints on emergent 1-vs-1 interpersonal patterns of play in football. Human Movement Science, 54, 347-353.

Mitchell, S. A., Oslin, J. L., \& Griffin, L. L. (2013). Teaching sport concepts and skills: A tactical games approach for ages 7 to 18. Human Kinetics.

Olthof, S. B., Frencken, W. G., \& Lemmink, K. A. (2015). The older, the wider: On-field tactical behavior of elite-standard youth soccer players in small-sided games. Human Movement Science, 41, 92-102. http://dx.doi.org/10.1016/j.humov.2015.02.004.

Pincus, S. M. (1991). Approximate entropy as a measure of system complexity. PNAS, 88(6), 2297-2301. http://dx.doi.org/10.1073/pnas.88.6.2297.

Sampaio, J., Lago, C., Gonçalves, B., Maçãs, V., \& Leite, N. (2014). Effects of pacing, status and unbalance in time motion variables, heart rate and tactical behaviour when playing 5-a-side football small-sided games. Journal of Science and Medicine in Sport, 17(2), $229-233$.

Sampaio, J., \& Macas, V. (2012). Measuring tactical behaviour in football. International Journal of Sports Medicine, 33(5), 395-401. http://dx.doi.org/10.1055/s-00311301320.

Seifert, L., Button, C., \& Davids, K. (2013). Key properties of expert movement systems in sport: An ecological dynamics perspective. Sports Medicine, 43(3), 167-178. http://dx.doi.org/10.1007/s40279-012-0011-z.

Serra-Olivares, J., González-Víllora, S., García-López, L. M., \& Araújo, D. (2015). Game-based approaches' pedagogical principles: Exploring task constraints in youth soccer. Journal of Human Kinetics, 46(1), 251-261.

Sgrò, F., Bracco, S., Pignato, S., \& Lipoma, M. (2018). Small-sided games and technical skills in soccer training: Systematic review and implications for sport and physical education practitioners. Journal of Sports Science, 6, 9-19.

Silva, P., Duarte, R., Sampaio, J., Aguiar, P., Davids, K., Araujo, D., \& Garganta, J. (2014). Field dimension and skill level constrain team tactical behaviours in smallsided and conditioned games in football. Journal of Sports Sciences, 32(20), 1888-1896. http://dx.doi.org/10.1080/02640414.2014.961950.

Stergiou, N., Buzzi, U., Kurz, M., \& Heidel, J. (2004). Nonlinear tools in human movement. In N. Stergiou (Ed.). Innovative analyses of human movement (pp. 63-90). Champaign, IL: Human Kinetics.

Travassos, B., Araujo, D., Duarte, R., \& McGarry, T. (2012). Spatiotemporal coordination behaviors in futsal (indoor football) are guided by informational game constraints. Human Movement Science, 31(4), 932-945. http://dx.doi.org/10.1016/j.humov.2011.10.004.

Travassos, B., Duarte, R., Vilar, L., Davids, K., \& Araújo, D. (2012). Practice task design in team sports: Representativeness enhanced by increasing opportunities for action. Journal of Sports Sciences, 30(13), 1447-1454. http://dx.doi.org/10.1080/02640414.2012.712716.

Travassos, B., Gonçalves, B., Marcelino, R., Monteiro, R., \& Sampaio, J. (2014). How perceiving additional targets modifies teams' tactical behavior during football small-sided games. Human Movement Science, 38, 241-250.

Vilar, L., Araujo, D., Travassos, B., \& Davids, K. (2014). Coordination tendencies are shaped by attacker and defender interactions with the goal and the ball in futsal. Human Movement Science, 33, 14-24. http://dx.doi.org/10.1016/j.humov.2013.08.012.

Vilar, L., Duarte, R., Silva, P., Chow, J. Y., \& Davids, K. (2014). The influence of pitch dimensions on performance during small-sided and conditioned soccer games. Journal of Sports Sciences, 32(19), 1751-1759. http://dx.doi.org/10.1080/02640414.2014.918640. 